\title{
Dynamic Integration of Regression Models
}

\author{
Niall Rooney ${ }^{1}$, David Patterson ${ }^{1}$, Sarab Anand $^{1}$, Alexey Tsymbal $^{2}$ \\ ${ }^{1}$ NIKEL, Faculty of Engineering, 16J27 \\ University Of Ulster at Jordanstown \\ Newtonabbey, BT37 OQB, United Kingdom \\ \{nf.rooney, wd.patterson, ss.anand\}@ulster.ac.uk \\ ${ }^{2}$ Alexey Tsymbal, Department Of Computer Science, Trinity College Dublin \\ \{Alexey.Tsymbal\}@cs.tcd.de
}

\begin{abstract}
In this paper we adapt the recently proposed Dynamic Integration ensemble techniques for regression problems and compare their performance to the base models and to the popular ensemble technique of Stacked Regression. We show that the Dynamic Integration techniques are as effective for regression as Stacked Regression when the base models are simple. In addition, we demonstrate an extension to both Stacked Regression and Dynamic Integration to reduce the ensemble set in size and assess its effectiveness.
\end{abstract}

\section{Introduction}

The purpose of ensemble learning is to build a learning model which integrates a number of base learning models, so that the model gives better generalization performance on application to a particular data-set than any of the individual base models [3]. Ensemble learning consists of two problems; ensemble generation: how does one generate appropriate base models? and ensemble integration: how does one integrate the base models' predictions to improve performance? Ensemble generation can be characterized as being homogeneous if each base learning model uses the same learning algorithm or heterogeneous if the base models can be built from a range of learning algorithms. Ensemble integration can be addressed by either one of two mechanisms, either the predictions of the base models are combined in some fashion during the application phase to give an ensemble prediction (combination/fusion approach) or the prediction of one base model is selected according to some criteria to form the final prediction (selection approach) [9].

Theoretical and empirical work has shown the ensemble approach to be effective with the proviso that the base models are diverse and sufficiently accurate [3]. These measures are however not necessarily independent of each other. If the prediction error of all base models is very low, then their learning hypothesis must be very similar to the true function underlying the data, and hence they must of necessity, be similar to each other i.e. they are unlikely to be diverse. In essence then there is often a trade-off between diversity and accuracy [2].

There has been much research work on ensemble learning for regression in the context of neural networks, however there has been less research carried out in terms 
of using homogeneous ensemble techniques to improve the performance of simple regression algorithms. In this paper we look at improving the generalization performance of nearest neighbours (k-NN) and least squares linear regression (LR). These methods were chosen as they are simple models with different approaches to learning in that linear regression is an eager model which tries to approximate the true function by a global linear function and k-nearest neighbours is a lazy model which tries to approximate the true function locally.

\section{Ensemble Integration}

The initial approaches to ensemble combination for regression were based on the linear combination of the base models according to the function:

$$
\sum_{i=1}^{n} \alpha_{i} f_{i}(x)
$$

where $\alpha_{i}$ is the weight assigned to the base models prediction $f_{i}(x)$. The simplest approach to determining the values of $\alpha_{i}$ is to set them to the same value. This is known as the Base Ensemble Method (BEM). More advanced approaches try to set the weights so as to minimize the mean square error of the training data. Merz and Pazzani [12] provide an extensive description of these techniques

Model selection simply chooses the best "base" model to make a prediction. This can be either done in a static fashion using cross validation majority [15] where the best model is the one that has the lowest training error. Alternatively it can be done in a dynamic fashion $[4,11,13]$ where based on finding "close" instances in the training data to a test instance, a base model is chosen which according to certain criteria is believed will give the best prediction. The advantage of this approach is based on the rationale that one model may perform better than other learning models in a localised region of the instance space even if, on average over the whole instance space, it performs no better than the others.

An alternative strategy to model integration is to build a meta-model to select/combine the outputs from base models. The original and most widely used metatechnique is referred to Stacking. Stacking was introduced by Wolpert [18] and was shown theoretically by LeBlanc and Tibshirani [9] to be a bias reducing technique. In Stacked Regression (SR), the base models produce meta-instances consisting of the target value and the base models' predictions, created by running cross validation over the training data. The meta-data is used to build a meta-model, based on a regression algorithm and the base models are built using the whole training data. Ensemble prediction for a test instance is formed by passing a meta test instance (formed from the base models' predictions) to the meta-model. Typically the generation of the base models is heterogeneous or homogeneous but built with different training parameters. Breiman [1] investigated the use of Linear Regression to form the meta-model and 
found that Linear Regression is a suitable meta-model so long as the coefficients of regression are constrained to be non-negative.

More recent meta-approaches for classification are the Dynamic Integration techniques developed by Puuronen and Tsymbal $[13,16]$ Similar to Stacking, these perform a cross-validation history during the training phase. However meta-instances are formed consisting of the training instance attribute values and the error for each model in predicting its target value. During the test phase a lazy meta-model based on weighted nearest neighbours uses the meta-data to either dynamically select or combine models for a test instance in the application phase. In the Methodology section we describe in detail the DI techniques and the modifications required to make them applicable for regression. In this paper, we compare the accuracy of ensemble techniques of SR and DI over a range of data-sets. It is particularly apposite to compare SR to the variants of DI as there strong similarities in their approach in that they accumulate meta-data based on a cross validation history which is then used to build a meta-model.

\section{Methodology}

In this section we describe the DI classification algorithms and their regression variants. DI consists of 3 techniques Dynamic Selection, Dynamic Voting and Dynamic Voting with Selection. We refer to their regression counterparts as Dynamic Selection, Dynamic Weighting and Dynamic Weighting with Selection. Dynamic Selection makes a localized selection of a model based on which model has the lowest cumulative error for the nearest neighbours to the test instance. The procedure for regression remains the same. Dynamic Voting assigns a weight to each base model based on its localized performance on the NN set and the final classification is based on weighted voting. Dynamic Weighting (DW) is similar to the Dynamic Voting in its calculation of weights but the final prediction is made by summing each of the base models predictions weighted by a normalized weight value. Dynamic Weighting with Selection (DWS) is a regression derivative of Dynamic Voting with Selection. The process is similar to Dynamic Weighting except that base model with cumulative error in the upper half of the error interval, $E_{i}>\left(E^{\max }-E^{\min }\right) / 2$, (where $E^{\max }$ is the largest cumulative error of any model and $E^{\mathrm{min}}$ is the lowest cumulative error of any model ) are discarded from adding to the prediction.

In [16] the ensemble generation is improved upon by a feature selection method based on hill climbing. In this paper, we consider a method that tries to reduce the size of the ensemble set whilst maintaining its accuracy. During the training phase, we start with a set of $N$ base models, and due to a consideration of their training accuracy and diversity determined by the cross-validation process (intrinsic to both the SR and the DI techniques) reduce the size of the set down to $M$ base models. This process of filtering down the number of models is based on the pseudo-code described in Figure 1 and adds little algorithmic overhead to the techniques. Its goal is to remove 
members from the ensemble that are considered too inaccurate to be effective and then to consider the remaining members based on both their accuracy and diversity.

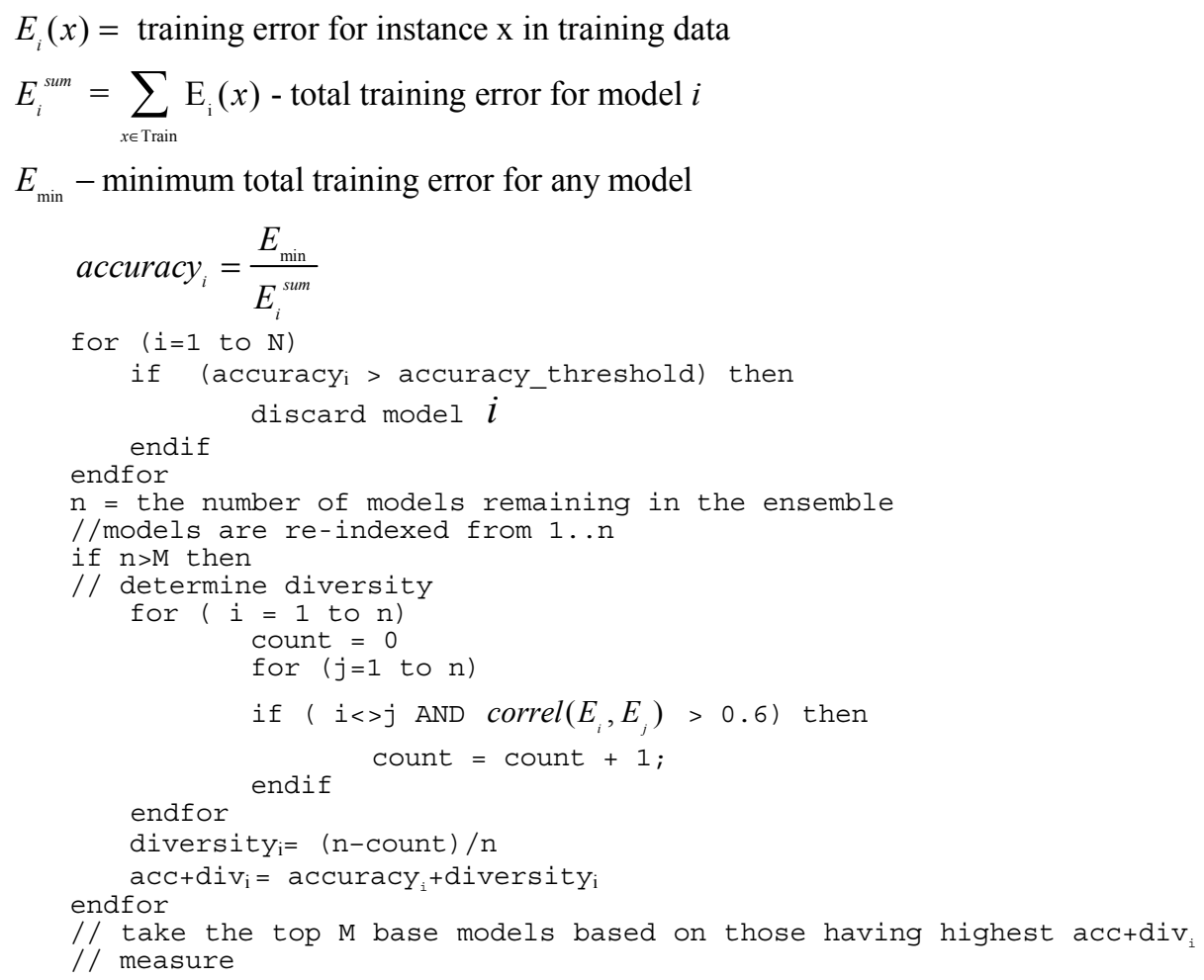

Figure 1 Ensemble size reduction technique

\section{Experimental Setup}

The base models and ensemble techniques were assessed using 10 fold cross validation and the mean absolute error (MAE) was recorded for each technique. 15 data-sets were chosen from the WEKA repository [18]. The data-sets were chosen as they represent real world data and not artificial regression examples. The data-sets were preprocessed to remove missing values using a mean or modal technique. The two base models used were 5-NN and Linear Regression. We assessed the improvement in accuracy or otherwise of the ensemble in comparison to the base model by using a two tailed paired t-test $(\mathrm{p}=0.05)$. For each technique, the ensemble set was generated using the Random sub-space method (RSM) first proposed by Ho [5,6] for classification problems and is a derivative of Stochastic Discrimination [7]. Random sub-space method is a generation method where each base model is built from the training data 
which has been transformed to contain different random subsets of the variables. We chose the model tree technique M5, which combines instance based learning with regression trees [14] as the meta-model for SR. We chose this as it has a larger hypothesis space than simple linear regression. In the experiments where the ensemble size was reduced the initial ensemble set had size $N=25$ and was reduced to $M=10$ with an accuracy_threshold of 0.66 . Each of the DI techniques used distance weighted 5-NN as their meta-model.

\section{Experimental Results}

This section is divided into two sections where each section consists of two experiments; the first is related to the accuracy of the ensembles for the whole ensemble set; the second assesses the accuracy of the ensemble to the experiments when the ensembles are reduced in size. Each section consists of the results of the comparison with the base model of LR and 5-NN respectively. The results of each experiment over the 15 data-sets is presented in the form of a table where the first column gives the name of the data-set, the second column the base models' $\mathrm{MAE} \pm$ standard deviation for each data-set, and column 3-6 gives the MAE for each ensemble technique. The remaining column records the technique with the least MAE, if any of the techniques were able to significantly improve upon the performance of the base model, otherwise the entry is left blank. An ensemble MAE result which is significantly better than the base model is shown in bold, if it is significantly worse it is shown underlined. An adjunct table summarizes the results of the significance comparison in the form of wins/ draws/losses where wins is the number of data-sets where the ensemble outperformed the base model, draws is the number of data-sets for which the base model showed no significant difference in accuracy to the base model, and losses is the number of datasets where the ensemble accuracy was worse than the base model.

\subsection{Whole Ensemble set}

This section refers to experimental results involving the whole ensemble set. Table 1 shows the results of the comparison when the base model was Linear Regression. DS and DWS reduced the error significantly for the greatest number of data-sets whereas DW reduced the error for the least number. However both SR and DS increased MAE significantly for two data-sets. Only DWS never increased the MAE significantly for any of the 15-data-sets. If one considers the "least MAE" column it is clear than for 7 data-sets none of the techniques were effective. For the other 8 data-sets, if we rank the order in which the ensemble technique gave the least error most frequently, DS came first with SR second. 


\begin{tabular}{|l|l|l|l|l|l|l|}
\hline Data-set & \multicolumn{1}{|c|}{ LR } & \multicolumn{1}{|c|}{ SR } & \multicolumn{1}{|c|}{ DS } & \multicolumn{1}{|c|}{ DW } & \multicolumn{1}{|c|}{ DWS } & $\begin{array}{l}\text { Least } \\
\text { MAE }\end{array}$ \\
\hline abalone & $1.58 \pm 0.08$ & $1.61 \pm 0.28$ & $1.58 \pm 0.09$ & $1.62 \pm 0.11$ & $1.58 \pm 0.10$ & - \\
\hline autohorse & $7.99 \pm 4.17$ & $\mathbf{6 . 5 4} \pm \mathbf{4 . 1 1}$ & $7.18 \pm 5.16$ & $\mathbf{6 . 8 4} \pm \mathbf{4 . 1 3}$ & $\mathbf{6 . 6 5} \pm \mathbf{4 . 2 3}$ & SR \\
\hline autoMpg & $2.23 \pm 0.21$ & $2.11 \pm 0.48$ & $\mathbf{2 . 0 5} \pm \mathbf{0 . 2 6}$ & $2.16 \pm 0.21$ & $\mathbf{2 . 0 5} \pm \mathbf{0 . 2 5}$ & DS/DWS \\
\hline autoPrice & $1974.23 \pm$ & $\mathbf{1 6 5 9 . 3 3} \pm$ & $\mathbf{1 5 1 8 . 5 8} \pm$ & $\mathbf{1 6 6 0 . 2 9} \pm$ & $\mathbf{1 5 3 2 . 6 5} \pm$ & DS \\
& 326.81 & $\mathbf{2 9 0 . 6 8}$ & $\mathbf{3 3 9 . 9 6}$ & $\mathbf{3 6 7 . 2 8}$ & $\mathbf{3 5 7 . 1}$ & \\
\hline auto93 & $3.79 \pm 1.3$ & $4.11 \pm 1.6$ & $4.02 \pm 1.17$ & $3.20 \pm 1.23$ & $3.25 \pm 1.28$ & - \\
\hline bodyfat & $0.53 \pm 0.23$ & $0.53 \pm 0.22$ & $\mathbf{0 . 4 3} \pm \mathbf{0 . 2 6}$ & $\underline{0.60 \pm 0.21}$ & $\mathbf{0 . 4 8} \pm \mathbf{0 . 2 4}$ & DS \\
\hline breastTumor & $7.97 \pm 1.05$ & $8.1 \pm 1.05$ & $8.06 \pm 0.99$ & $7.77 \pm 0.93$ & $7.84 \pm 0.89$ & - \\
\hline cholesterol & $39.24 \pm 5.88$ & $\underline{40.89 \pm 5.73}$ & $38.92 \pm 4.64$ & $38.19 \pm 4.62$ & $38.41 \pm 4.44$ & - \\
\hline cloud & $0.26 \pm 0.09$ & $0.26 \pm 0.09$ & $\underline{0.32 \pm 0.08}$ & $0.27 \pm 0.09$ & $0.26 \pm 0.09$ & - \\
\hline cpu & $35.02 \pm 4.45$ & $\mathbf{1 4 . 2 2} \pm \mathbf{6 . 7 5}$ & $\mathbf{2 2 . 2 5} \pm 7.31$ & $\mathbf{2 1 . 2 4} \pm \mathbf{8 . 0 5}$ & $\mathbf{1 9 . 3 6} \pm \mathbf{7 . 1 3}$ & SR \\
\hline housing & $3.41 \pm 0.33$ & $\mathbf{2 . 8 2} \pm \mathbf{0 . 5 8}$ & $\mathbf{2 . 6 8} \pm \mathbf{0 . 4 7}$ & $3.29 \pm 0.56$ & $\mathbf{2 . 9 6} \pm \mathbf{0 . 5 4}$ & DS \\
\hline lowbwt & $364.48 \pm$ & $\underline{392.01 \pm}$ & $\underline{397.93 \pm}$ & $356.87 \pm$ & $363.03 \pm$ & - \\
& 48.21 & $\underline{\mathbf{5 7 . 4}}$ & $\underline{\mathbf{5 1 . 4 8}}$ & 62.64 & 61.44 & \\
\hline sensory & $0.61 \pm 0.04$ & $0.61 \pm 0.04$ & $\mathbf{0 . 5 9} \pm \mathbf{0 . 0 5}$ & $0.61 \pm 0.06$ & $0.59 \pm 0.06$ & - \\
\hline servo & $0.63 \pm 0.273$ & $\mathbf{0 . 3 8} \pm \mathbf{0 . 2 3}$ & $\mathbf{0 . 4 5} \pm \mathbf{0 . 2 8}$ & $0.63 \pm 0.22$ & $\mathbf{0 . 4 4} \pm \mathbf{0 . 2 5}$ & SR \\
\hline strike & $221.43 \pm$ & $\mathbf{2 0 9 . 7 9} \pm$ & $\mathbf{1 8 0 . 8 4} \pm$ & $\mathbf{2 0 3 . 6 4} \pm \mathbf{3 8 . 3 3}$ & $\mathbf{1 8 9 . 0 1} \pm$ & DS \\
& 38.47 & $\mathbf{4 1 . 6 5}$ & $\mathbf{4 5 . 7 2}$ & & $\mathbf{4 2 . 5 4}$ & \\
\hline
\end{tabular}

\begin{tabular}{|l|l|l|l|l|}
\hline Method & SR & DS & DW & DWS \\
\hline Wins/Ties /Losses & $6 / 7 / 2$ & $8 / 5 / 2$ & $4 / 9 / 2$ & $8 / 7 / 0$ \\
\hline
\end{tabular}

Table 1. The comparison of ensembles using LR as the base model

\begin{tabular}{|l|l|l|l|l|l|l|}
\hline Data-set & \multicolumn{1}{|c|}{$\mathbf{5 - N N}$} & \multicolumn{1}{|c|}{ SR } & \multicolumn{1}{|c|}{ DS } & \multicolumn{1}{|c|}{ DW } & \multicolumn{1}{|c|}{ DWS } & Least MAE \\
\hline Abalone & $1.61 \pm 0.09$ & $\mathbf{1 . 5 4} \pm \mathbf{0 . 0 8}$ & $\underline{1.73 \pm 0.07}$ & $\mathbf{1 . 5 4} \pm \mathbf{0 . 0 9}$ & $\mathbf{1 . 5 4} \pm \mathbf{0 . 0 9}$ & $S R / D W / D W S$ \\
\hline autohorse & $8.7 \pm 4.69$ & $\mathbf{7 . 1 1} \pm \mathbf{3 . 7 1}$ & $\mathbf{5 . 7 9} \pm \mathbf{4 . 5 7}$ & $\mathbf{6 . 4 4} \pm \mathbf{4 . 9 3}$ & $\mathbf{6 . 0 6} \pm \mathbf{4 . 9 2}$ & $D S$ \\
\hline autompg & $2.31 \pm 0.38$ & $\mathbf{2 . 1 2} \pm \mathbf{0 . 3 4}$ & $2.41 \pm 0.35$ & $\mathbf{2 . 0 4} \pm \mathbf{0 . 3 5}$ & $\mathbf{2 . 0 8} \pm \mathbf{0 . 3 5}$ & $D W$ \\
\hline autoprice & $1531.86 \pm$ & $1478.62 \pm$ & $1382.06 \pm$ & $\mathbf{1 4 3 8 . 3 9} \pm$ & $\mathbf{1 3 9 7 . 6 3} \pm$ & $D W S$ \\
& 404.24 & 460.89 & 336.79 & $\mathbf{4 6 0 . 4 8}$ & $\mathbf{4 5 4 . 4 3}$ & \\
\hline auto93 & $3.81 \pm 1.4$ & $\underline{3.76 \pm 1.11}$ & $4.27 \pm 1.32$ & $\mathbf{3 . 4} \pm \mathbf{1 . 5 2}$ & $\mathbf{3 . 3 9} \pm \mathbf{1 . 5 8}$ & $D W S$ \\
\hline bodyfat & $2.3 \pm 0.49$ & $\mathbf{0 . 9 4} \pm \mathbf{0 . 2 1}$ & $\mathbf{1 . 1 6} \pm \mathbf{0 . 2 8}$ & $\mathbf{1 . 7} \pm \mathbf{0 . 3 7}$ & $\mathbf{1 . 4 0 7} \pm \mathbf{0 . 3 4}$ & $S R$ \\
\hline breastTumor & $9.39 \pm 1.04$ & $\mathbf{8 . 3 8} \pm \mathbf{0 . 6 4}$ & $9.67 \pm 1.06$ & $\mathbf{8 . 0 1} \pm \mathbf{0 . 9 1}$ & $\mathbf{8 . 1 2} \pm \mathbf{0 . 9 7}$ & $D W$ \\
\hline cholesterol & $43.0 \pm 4.13$ & $43.39 \pm 4.03$ & $46.17 \pm 6.04$ & $\mathbf{3 9 . 6 4} \pm \mathbf{4 . 6 3}$ & $\mathbf{4 0 . 3 6} \pm \mathbf{4 . 5 6}$ & $D W$ \\
\hline cloud & $0.51 \pm 0.19$ & $\mathbf{0 . 3 8} \pm \mathbf{0 . 1 3}$ & $\mathbf{0 . 3 9} \pm \mathbf{0 . 1 1}$ & $\mathbf{0 . 3 9} \pm \mathbf{0 . 1 7}$ & $\mathbf{0 . 3 6} \pm \mathbf{0 . 1 4}$ & $D W S$ \\
\hline Cpu & $22.72 \pm 13.94$ & $\underline{34.16 \pm 17.61}$ & $23.97 \pm 12.97$ & $\mathbf{1 9 . 6 8} \pm \mathbf{1 3 . 4 6}$ & $20.72 \pm 14.18$ & $D W$ \\
\hline housing & $2.59 \pm 0.58$ & $\mathbf{2 . 3 0} \pm \mathbf{0 . 4 1}$ & $2.56 \pm 0.39$ & $\mathbf{2 . 3 9} \pm \mathbf{0 . 5 5}$ & $\mathbf{2 . 2 7} \pm \mathbf{0 . 5}$ & $D W S$ \\
\hline lowbwt & $398.3 \pm 80.6$ & $397.8 \pm 47.17$ & $\underline{471.35 \pm}$ & $365.88 \pm$ & $369.47 \pm$ & $D W S$ \\
& & & $\underline{67.56}$ & 80.53 & 74.71 & \\
\hline sensory & $0.6 \pm 0.06$ & $\mathbf{0 . 5 5} \pm \mathbf{0 . 0 6}$ & $\underline{0.66 \pm 0.07}$ & $\mathbf{0 . 5 8} \pm \mathbf{0 . 0 5}$ & $\mathbf{0 . 5 8} \pm \mathbf{0 . 0 5}$ & $S R$ \\
\hline servo & $0.56 \pm 0.19$ & $\mathbf{0 . 3 8} \pm \mathbf{0 . 3 0}$ & $\mathbf{0 . 4 2} \pm \mathbf{0 . 2 4}$ & $0.62 \pm 0.22$ & $\mathbf{0 . 4 2} \pm \mathbf{0 . 2 2}$ & $S R$ \\
\hline strike & $194.62 \pm$ & $\underline{222.29 \pm}$ & $196.71 \pm$ & $\mathbf{1 8 2 . 2 5} \pm$ & $\mathbf{1 7 6 . 0 8} \pm$ & $D W S$ \\
& 53.46 & $\underline{46.7}$ & 50.16 & $\mathbf{5 0 . 0 1}$ & $\mathbf{5 0 . 1 5}$ & \\
\hline
\end{tabular}

\begin{tabular}{|l|l|l|l|l|}
\hline Method & SR & DS & DW & DWS \\
\hline Wins/ Draws/losses & $9 / 3 / 3$ & $4 / 7 / 4$ & $13 / 2 / 0$ & $13 / 2 / 0$ \\
\hline
\end{tabular}

Table 2. The comparison of ensembles using $5-\mathrm{NN}$ as the base model

Table 2 shows the results of the comparison of ensembles when the base model was 5-NN. Clearly the two outstanding ensemble techniques were DW and DWS, which both reduced the error signicantly for 13 out of the 15 data-sets. The technique which proved least effective was DS. The "least error" column shows that for every data-set at least one of the ensemble techniques was effective in signifcantly reducing 
the error. DWS came first in rank order of the techniques which gave the least error most frequently with DW coming second.

In summary, it can be seen that for either base model, at least one of the DI techniques is as effective as SR, if not more so in reducing the error. Also DWS seemed to be the most reliable ensemble approach, as it never significantly increased the error. The pattern of behaviour of the DI techniques for regression mirrors that of classification [16] where the best integration method varied with the data-set and the base model.

\subsection{Reduced Ensemble set}

In this section, we repeated the experiments of the previous section, but with the addition that the ensemble set had been reduced at the end of the training phase using the algorithm described in Figure 1 from $N=25$ to $M=10$. Table 3 shows the results of the comparison of the reduced size ensembles for LR. Comparing the ties/wins/losses of Table 3 to Table 1 shows that DW and DS improved in performance, DWS remained the same and SR remained approximately the same.

\begin{tabular}{|l|l|l|l|l|l|}
\hline Data-set & \multicolumn{1}{|c|}{ LR } & \multicolumn{1}{|c|}{ SR } & \multicolumn{1}{|c|}{ DS } & \multicolumn{1}{|c|}{ DW } & \multicolumn{1}{c|}{ DWS } \\
\hline abalone & $1.58 \pm 0.08$ & $\mathbf{1 . 5 2} \pm \mathbf{0 . 0 6}$ & $1.57 \pm 0.09$ & $1.60 \pm 0.09$ & $1.58 \pm 0.09$ \\
\hline autohorse & $7.99 \pm 4.17$ & $7.42 \pm 3.4$ & $7.00 \pm 4.75$ & $\mathbf{6 . 5 7} \pm \mathbf{3 . 6 9}$ & $\mathbf{6 . 2} \pm \mathbf{3 . 8 7}$ \\
\hline autompg & $2.23 \pm 0.21$ & $2.09 \pm 0.43$ & $\mathbf{2 . 0 9} \pm \mathbf{0 . 3 3}$ & $\mathbf{2 . 1 3} \pm \mathbf{0 . 2}$ & $\mathbf{2 . 0 8} \pm \mathbf{0 . 2 4}$ \\
\hline autoPrice & $1974.23 \pm$ & $\mathbf{1 6 8 7 . 6 8} \pm$ & $\mathbf{1 5 5 0 . 6 1} \pm$ & $\mathbf{1 7 2 3 . 9 9} \pm$ & $\mathbf{1 5 6 7 . 5 5} \pm$ \\
& 326.81 & $\mathbf{2 3 3 . 3 7}$ & $\mathbf{3 4 3 . 3 2}$ & $\mathbf{3 2 4 . 9 6}$ & $\mathbf{3 5 6 . 5 6}$ \\
\hline auto93 & $3.79 \pm 1.3$ & $3.521 \pm 1.1$ & $3.91 \pm 1.24$ & $3.43 \pm 1.33$ & $3.41 \pm 1.39$ \\
\hline bodyfat & $0.53 \pm 0.23$ & $0.48 \pm 0.23$ & $\mathbf{0 . 4 1} \pm \mathbf{0 . 2 7}$ & $\mathbf{0 . 4 5} \pm \mathbf{0 . 2 5}$ & $\mathbf{0 . 4 2} \pm \mathbf{0 . 2 6}$ \\
\hline breastTumor & $7.97 \pm 1.05$ & $8.08 \pm 1.09$ & $8.06 \pm 0.99$ & $7.92 \pm 0.95$ & $7.97 \pm 0.89$ \\
\hline cholesterol & $39.24 \pm 5.88$ & $38.94 \pm 5.76$ & $39.01 \pm 4.96$ & $39.05 \pm 4.63$ & $39.03 \pm 4.49$ \\
\hline cloud & $0.26 \pm 0.09$ & $0.28 \pm 0.09$ & $0.28 \pm 0.10$ & $0.27 \pm 0.10$ & $0.27 \pm 0.10$ \\
\hline cpu & $35.02 \pm 4.45$ & $\mathbf{1 5 . 3 5} \pm \mathbf{6 . 8}$ & $\mathbf{2 4 . 2 7} \pm \mathbf{6 . 0 1}$ & $\mathbf{2 5 . 0 5} \pm 7.2$ & $\mathbf{2 3 . 0 7} \pm 7.09$ \\
\hline housing & $3.41 \pm 0.33$ & $\mathbf{2 . 7 6} \pm \mathbf{0 . 3 7}$ & $\mathbf{2 . 6 7} \pm \mathbf{0 . 4 5}$ & $3.17 \pm 0.42$ & $\mathbf{2 . 7 9} \pm \mathbf{0 . 4 7}$ \\
\hline lowbwt & $364.48 \pm 48.21$ & $365.46 \pm 50.86$ & $376.69 \pm 42.86$ & $365.19 \pm 44.73$ & $361.59 \pm 49.06$ \\
\hline sensory & $0.61 \pm 0.04$ & $0.61 \pm 0.04$ & $\mathbf{0 . 5 9} \pm \mathbf{0 . 0 4}$ & $0.60 \pm 0.05$ & $0.59 \pm 0.05$ \\
\hline Servo & $0.63 \pm 0.27$ & $\mathbf{0 . 4 4} \pm \mathbf{0 . 2}$ & $\mathbf{0 . 5} \pm \mathbf{0 . 2 3}$ & $\mathbf{0 . 5 3} \pm \mathbf{0 . 2 7}$ & $\mathbf{0 . 4 8} \pm \mathbf{0 . 2 7}$ \\
\hline Strike & $221.43 \pm 38.47$ & $218.47 \pm 37.39$ & $\mathbf{2 0 5 . 0 4} \pm \mathbf{3 6 . 6 8}$ & $\mathbf{2 1 2 . 6 2} \pm \mathbf{3 3 . 9 9}$ & $\mathbf{2 0 5 . 3 9} \pm \mathbf{3 5 . 3 5}$ \\
\hline
\end{tabular}

\begin{tabular}{|l|l|l|l|l|}
\hline Method & SR & DS & DW & DWS \\
\hline Wins/ Ties/losses & $5 / 10 / 0$ & $8 / 7 / 0$ & $7 / 8 / 0$ & $8 / 7 / 0$ \\
\hline
\end{tabular}

Table 3. Results of comparison of ensembles using LR

There is however more variation in the results than the summary in significance comparison alone would suggest. If we calculate the percentage change in MAE between the results in Table 1 and Table 3 and average it over all data-sets, the following average percentage changes are shown in Table 4. A positive value is recorded if the technique gave on average a percentage reduction in error.

It is clear that although the average change in MAE is quite small no larger than a $2 \%$ decrease , the standard deviation is relatively large indicating that for some datasets there is a large percentage change in the MAE. 


\begin{tabular}{|l|c|c|c|c|}
\hline Technique & SR & DS & DW & DWS \\
\hline $\begin{array}{l}\text { Average percentage change in } \\
\text { MAE }\end{array}$ & $-0.45 \pm 8.3$ & $-0.72 \pm 6.36$ & $0.9 \pm 9.89$ & $-1.41 \pm 7.41$ \\
\hline
\end{tabular}

Table 4. Percentage change in MAE for ensemble size from $\mathrm{N}$ to $\mathrm{M}$

However comparing the reduced ensemble set to the whole ensemble results in detail shows a general trend that for data-sets where the error increased it did not increase to change the level of significance, but where the error decreased then in some cases it did change the signifcance comparison. e.g. consider the technique DW, for the whole ensemble set, autohorse, autoprice, cpu, strike gave an MAE better than the base model whereas abalone, and bodyfat were significantly worse. For the reduced ensemble set, autohorse, autompg, autoprice, bodyfat, cpu, servo, strike gave an MAE significantly better than base model even though for some of these data-sets there was a relative increase in MAE.

\begin{tabular}{|l|l|l|l|l|l|}
\hline Data-set & \multicolumn{1}{|c|}{$5-\mathrm{NN}$} & \multicolumn{1}{|c|}{$\mathrm{SR}$} & \multicolumn{1}{c|}{$\mathrm{DS}$} & \multicolumn{1}{c|}{$\mathrm{DW}$} & \multicolumn{1}{c|}{ DWS } \\
\hline abalone & $1.61 \pm 0.09$ & $\mathbf{1 . 5 6} \pm \mathbf{0 . 0 9}$ & $\underline{1.756 \pm 0.07}$ & $\mathbf{1 . 5 7} \pm \mathbf{0 . 0 9}$ & $1.589 \pm 0.09$ \\
\hline autohorse & $8.7 \pm 4.69$ & $\mathbf{6 . 1 4} \pm \mathbf{3 . 7 1}$ & $\mathbf{4 . 8 8} \pm \mathbf{4 . 9 2}$ & $\mathbf{4 . 7 6} \pm \mathbf{4 . 3 9}$ & $\mathbf{4 . 7 7} \pm \mathbf{4 . 7 2}$ \\
\hline autoMpg & $2.31 \pm 0.38$ & $\mathbf{2 . 1 3} \pm \mathbf{0 . 3 4}$ & $2.34 \pm 0.39$ & $\mathbf{2 . 0 0} \pm \mathbf{0 . 3 9}$ & $\mathbf{2 . 0 6} \pm \mathbf{0 . 4 1}$ \\
\hline autoPrice & $1531.86 \pm$ & $1383.66 \pm$ & $\mathbf{1 3 2 0 . 4 1} \pm$ & $\mathbf{1 3 1 3 . 7 8} \pm$ & $\mathbf{1 3 2 6 . 4 1} \pm$ \\
& 404.24 & 460.89 & $\mathbf{2 3 6 . 9 1}$ & $\mathbf{4 6 2 . 2 8}$ & $\mathbf{4 1 9 . 8 1}$ \\
\hline auto93 & $3.81 \pm 1.4$ & $3.61 \pm 1.11$ & $4.28 \pm 1.29$ & $3.5 \pm 1.6$ & $3.56 \pm 1.58$ \\
\hline bodyfat & $2.3 \pm 0.49$ & $\mathbf{0 . 9 5} \pm \mathbf{0 . 2 1}$ & $\mathbf{1 . 0 7} \pm \mathbf{0 . 2 8}$ & $\mathbf{1 . 0 3} \pm \mathbf{0 . 3 2}$ & $\mathbf{1 . 0 4} \pm \mathbf{0 . 2 8}$ \\
\hline breastTumor & $9.39 \pm 1.04$ & $\mathbf{8 . 2 4} \pm \mathbf{0 . 6 4}$ & $9.71 \pm 0.99$ & $\mathbf{7 . 9 9} \pm \mathbf{0 . 9 1}$ & $\mathbf{8 . 3 2} \pm \mathbf{1 . 0}$ \\
\hline cholesterol & $43.0 \pm 4.13$ & $\mathbf{3 9 . 8 1} \pm \mathbf{4 . 0 3}$ & $46.77 \pm 6.89$ & $\mathbf{4 0 . 1 3} \pm \mathbf{4 . 7 2}$ & $41.21 \pm 4.77$ \\
\hline cloud & $0.51 \pm 0.19$ & $\mathbf{0 . 3 7} \pm \mathbf{0 . 1 3}$ & $0.37 \pm 0.10$ & $\mathbf{0 . 3 3} \pm \mathbf{0 . 1 4}$ & $\mathbf{0 . 3 4} \pm \mathbf{0 . 1 2}$ \\
\hline cpu & $22.72 \pm 13.94$ & $\underline{30.97 \pm 17.61}$ & $21.10 \pm 13.33$ & $\mathbf{2 0 . 1 2} \pm \mathbf{1 2 . 4 8}$ & $21.33 \pm 12.95$ \\
\hline housing & $2.59 \pm 0.58$ & $\mathbf{2 . 3 3} \pm \mathbf{0 . 4 1}$ & $2.57 \pm 0.5$ & $\mathbf{2 . 3 3} \pm \mathbf{0 . 4 8}$ & $\mathbf{2 . 2 4} \pm \mathbf{0 . 4 1}$ \\
\hline lowbwt & $398.3 \pm 80.6$ & $375.46 \pm 47.17$ & $430.79 \pm 83.64$ & $\mathbf{3 5 9 . 3 9} \pm \mathbf{5 8 . 6 5}$ & $\mathbf{3 6 7 . 6 6} \pm \mathbf{6 4 . 3 1}$ \\
\hline sensory & $0.6 \pm 0.06$ & $\mathbf{0 . 5 6} \pm \mathbf{0 . 0 6}$ & $\underline{0.64} \pm 0.08$ & $\mathbf{0 . 5 7} \pm \mathbf{0 . 0 5}$ & $0.58 \pm 0.06$ \\
\hline servo & $0.56 \pm 0.19$ & $\mathbf{0 . 3 8} \pm \mathbf{0 . 3}$ & $\mathbf{0 . 4 4} \pm \mathbf{0 . 2 8}$ & $\mathbf{0 . 4 5} \pm \mathbf{0 . 2 9}$ & $\mathbf{0 . 3 9} \pm \mathbf{0 . 3}$ \\
\hline strike & $194.62 \pm 53.46$ & $\underline{208.72 \pm 46.7}$ & $195.78 \pm 61.88$ & $\mathbf{1 8 7 . 1 4} \pm \mathbf{5 2 . 4 6}$ & $185.92 \pm 56.27$ \\
\hline
\end{tabular}

\begin{tabular}{|l|l|l|l|l|}
\hline Method & SR & DS & DW & DWS \\
\hline Ties/Wins/losses & $10 / 3 / 2$ & $4 / 9 / 3$ & $14 / 1 / 0$ & $9 / 6 / 0$ \\
\hline
\end{tabular}

Table 5 Comparison of Ensembles with the base model 5-NN

Table 5 shows the comparison of the reduced ensemble sets when the base model was 5-NN. Comparing the results to Table 2 shows that SR, DS, DWS performed slightly better with the reduced sets. DWS showed a drop of 4 from 13 to 9 data-sets showing a significant improvement in MAE. The average percentage change in MAE for the whole ensemble set and the reduced ensemble set for 5-NN is shown in Table 6. However for those 4 data-sets which were no longer significantly better with DWS than the base model, the percentage change in MAE was at most 5.6\%. The same pattern of average error change is similar to LR with a low average pecentage change but a higher level of variability in percentage change amongst the data-sets, as shown in Table 6. The main difference to the results for LR is that for all techniques there 
was a positive change in the average percentage change in error, with a relatively large change for DW.

\begin{tabular}{|l|c|c|c|c|}
\hline Technique & SR & DS & DW & DWS \\
\hline $\begin{array}{l}\text { Average percentage change in } \\
\text { MAE }\end{array}$ & $3.49 \pm 4.69$ & $3.53 \pm 5.61$ & $7.42 \pm 13.23$ & $3.04+9.12$ \\
\hline
\end{tabular}

Table 6 Percentage change in MAE for ensemble size $=\mathrm{N}$ to $\mathrm{M}$

In summary, the ensemble size reduction strategy maintains the effective-ness both of SR and the DI techniques. In the case DW, the results would suggest that in fact pruning the ensemble set actually improves accuracy, a likely consequence that it more sensitive to in-accurate or redundant base models, than the DS and DWS approaches, which either select the best model or remove inaccurate models from the model combination.

\section{Conclusions and Future Work}

In this paper we have demonstrated that the classification ensemble techniques of Dynamic Integration can be adapted to the problem of regression. We have shown that for simple base models, these techniques are as effective as Stacked Regression for the range of data-sets tested. We have presented a extension to the SR and DI techniques which uses the accuracy and diversity measure captured in the training of the base models to prune the size of the ensemble thus removing models that are ineffective in the model combination. We intend to refine and improve on this simple technique as it provides little extra overhead to the algorithms and has shown promising results in reducing the ensemble size whilst maintaining its level of accuracy. In particular, we intend to investigate in more detail the appropriate choice of accuracy threshold and the size of the reduced ensemble set. Also, we shall compare our measure for diversity to the more commonly known measures for diversity such as the variance based measure developed in [8].

\section{Acknowledgements}

We gratefully acknowledge the WEKA framework [16] within which the ensemble methods were implemented and assessed. Dr Tsymbal acknowledges Science Foundation, Ireland.

\section{References}

1. Breiman, L. 1996. Stacked Regression. Machine Learning, 24:49-64. 
2. Christensen, S. 2003. Ensemble Construction via Designed Output Distortion In Proc. $4^{\text {th }}$ International Workshop on Multiple Classifier Systems, LNCS, Vol. 2709, pp. 286-295, Springer-Verlag.

3. Dietterich, T. 2000. Ensemble Methods in Machine Learning, In Proc. $1^{\text {st }}$ International Workshop on Multiple Classifer Systems, LNCS, Vol 1857, pp. 1-10, Springer-Verlag.

4. Giacinto, G. and Roli, F. 2000. Dynamic Classifier Selection, In Proc. $1^{\text {st }}$ Int. Workshop on Multiple Classifier Systems, LNCS, Vol 1857, pp. 177189, Springer-Verlag.

5. Ho, T. K. 1998a. The random subspace method for constructing decision forests. IEEE PAMI, 20(8):832--844.

6. Ho, T.K. 1998b. Nearest Neighbors in Random Subspaces, LNCS: Advances in Pattern Recognition, 640-648.

7. Kleinberg, E.M. 1990. Stochastic Discrimination, Annals of Mathematics and Artificial intelligence, 1:207-239.

8. Krogh, A. and Vedelsby, J. 1995. Neural Networks Ensembles, Cross validation, and Active Learning, Advances in Neutal Information Processing Systems, MIT Press, pp. 231-238.

9. Kuncheva L.I. 2002. Switching between selection and fusion in combining classifiers: An experiment, IEEE Transactions on SMC, Part B, 32 (2), 146156.

10. LeBlanc, M. and Tibshirani, R. 1992. Combining estimates in Regression and Classification, Technical Report, Dept. of Statistics, University of Toronto.

11. Merz, C.J. 1996. Dynamical selection of learning algorithms. In Learning from data, artificial intelligence and statistics. .Fisher and H.-J.Lenz (Eds.) New York: Springer.

12. Merz, C. and Pazzani, M. 1999. A principal components approach to combining regression estimates, Machine Learning, 36:9-32.

13. Puuronen, S., Terziyan, V., Tsymbal, A. 1999. A Dynamic Integration Algorithm for an Ensemble of Classifiers. Foundations of Intelligent Systems, 11th International Symposium ISMIS'99, LNAI, Vol. 1609: 592-600, Springer-Verlag.

14. Quinlan, R. 1992. Learning with continuous classes, In Proceedings of the $5^{\text {th }}$ Australian Joint Conference on Artificial Intelligence, World Scientific, pp. 343-348.

15. Schaffer, C. 1993. Overfitting avoidance as bias. Machine Learning 10:153178.

16. Sharkey, A.J C. (Ed.) 1999. Combining Artificial Neural Nets: Ensemble and Modular Multi-Net Systems. Springer-Verlag,.

17. Tsymbal, A., Puuronen, S., Patterson, D. 2003. Ensemble feature selection with the simple Bayesian classification, Information Fusion Vol. 4:87-100, Elsevier.

18. Witten, I. and Frank, E. 1999. Data Mining: Practical Machine Learning Tools and Techniques with Java Implementations, Morgan Kaufmann.

19. Wolpert, D. 1992. Stacked Generalization, Neural Networks 5, pp. 241-259. 